\title{
Searching atomic spin contrast on nickel oxide (001) by force microscopy
}

\author{
M. Schmid and J. Mannhart \\ Institute of Physics, Universität Augsburg, Electronic Correlations and Magnetism, Experimentalphysik VI, Universitätsstrasse 1, \\ D-86135 Augsburg, Germany \\ F. J. Giessibl* \\ Institute for Experimental and Applied Physics, Universität Regensburg, Universitätsstrasse 31, D-93040 Regensburg, Germany
}

(Received 21 March 2006; revised manuscript received 27 July 2007; published 2 January 2008)

\begin{abstract}
The (001) surface of $\mathrm{NiO}$, an antiferromagnet at room temperature, was investigated under ultrahigh vacuum conditions with frequency modulation atomic force microscopy. The antiferromagnetic coupling between ions leads to a spin superstructure on (001) surfaces. Exchange interaction between the probe of a force microscope and the $\mathrm{NiO}(001)$ surface should allow us to image spin superstructures in real space. The surface was imaged with three different probing tips: nonmagnetic W tips, ferromagnetic Co tips, and antiferromagnetic $\mathrm{NiO}$ tips-and atomic resolution was achieved with all three of them in various distance regimes and in several channels. Atomic resolution is obtained with all tips, but evidence for spin contrast is lacking although oscillation amplitudes in the angstrom regime have been used, where optimal signal-to-noise ratio is expected.
\end{abstract}

DOI: 10.1103/PhysRevB.77.045402

PACS number(s): 81.65.Cf, 81.65.Ps

\section{INTRODUCTION}

The electronic and mechanical properties of matter are dominated by the Coulomb interaction resulting from the charge of the electrons. In contrast, the magnetic interaction of the spin of the electrons plays a minor role. The dipoledipole interaction of single electronic spins for typical interatomic distances is only on the order of a few $\mu \mathrm{eV}$ whereas electrostatic energies between two electrons are $10^{6}$ times larger. While the direct interaction energy between spins is small, the Pauli principle constrains the symmetry of wave functions of two-electron states depending on spin: the spatial part of a spin-singlet state must keep its sign with particle exchange, while a spin-triplet state flips the sign of the spatial part of the wave function with particle exchange. In $\mathrm{H}_{2}$, the energetic difference between its two electrons occupying singlet vs triplet states (exchange interaction) amounts to several eV's. ${ }^{1}$ Considering the importance of spin in solids, it is essential to establish tools that allow us to analyze spin orientation on surfaces. For conductive samples, spinpolarized scanning tunneling microscopy ${ }^{2}$ is a powerful tool to image the spin orientation of surface atoms within magnetic domains or even antiferromagnetic surfaces with atomic resolution. ${ }^{3}$ Recently, the spin of a single magnetic ion placed on an insulating spacer over a metal surface has been measured by scanning tunneling spectroscopy. ${ }^{4}$ However, the spin orientation is also a key parameter controlling the physics of insulating bulk materials such as magnetic oxides. Insulators can be imaged by atomic force microscopy $(\mathrm{AFM}),{ }^{5}$ and magnetic force microscopy (MFM), a variation of AFM, allows magnetic imaging through the magnetic dipole interaction of magnetic domains in the probe tip and in the sample. Because of the weak dipole-dipole interaction, many spins comprising larger domains are necessary to measure magnetic dipole forces; thus, the spatial resolution of magnetic force microscopy is limited to some $10 \mathrm{~nm}$. In contrast, exchange interaction can lead to spin-dependent interaction energies of up to $100 \mathrm{meV}$. The feasibility of atomic imaging of exchange interactions (exchange force micros- copy) on ferromagnetic samples by AFM has been proposed by Nakamura et al. already in 1998 early after atomic resolution AFM became available. ${ }^{6}$ For two reasons, antiferromagnetic samples are attractive to probe the possibility of exchange force measurements: (a) they provide well-defined magnetic contrast over small lateral distances and (b) the disturbing magnetic dipole interaction between a magnetic tip and an antiferromagnetic sample is weak and decays exponentially with distance. $\mathrm{NiO}(001)$ is a good choice for a test sample, because it is antiferromagnetic at room temperature and (001) surfaces with excellent flatness and cleanliness can be prepared readily by cleavage in ultrahigh vacuum. Because of its magnetic properties, $\mathrm{NiO}$ is used as a pinning layer in spin valves and has been instrumental in the study of metal-insulator transitions. ${ }^{7} \mathrm{NiO}$ has been studied by elevated-temperature scanning tunneling microscopy. ${ }^{8}$ Several groups have studied $\mathrm{NiO}(001)$ by atomic force microscopy and obtained atomic images of the surface ${ }^{9-12}$ and performed spectroscopy, ${ }^{13-15}$ but a clear-cut proof of the expected spin contrast has been lacking until recently. ${ }^{16}$ In this experiment, Kaiser et al. provide a first experimental clue about the feasibility of exchange force microscopy that had been proposed almost a decade earlier. ${ }^{6}$ The authors operate a force microscope at liquid helium temperature and use a standard Si cantilever coated with a 22-nm-thick ferromagnetic iron film. Because of the small thickness of the magnetic coating of the cantilever tip, the magnetization of the tip is likely to be parallel to the surface. To induce a magnetization in the preferential direction normal to the sample surface, in Ref. 16 a $5 \mathrm{~T}$ external magnetic film has been applied to provide magnetic polarization of the tip.

Here, we strive to obtain spin contrast at room temperature without the need of an external magnetic field because it cannot be ruled out completely that the application of the magnetic field might induce structural changes in the $\mathrm{NiO}$ sample (magnetostriction) that interferes with the exchange contrast. Instead of using metal coated $\mathrm{Si}$ cantilevers as in Ref. 16, we use cantilevers that have mounted polycrystalline cobalt or single-crystal $\mathrm{NiO}$ tips as tips. These cantile- 


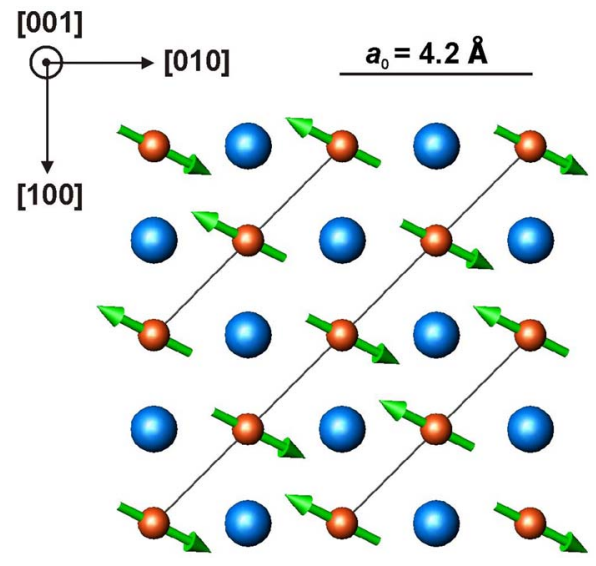

FIG. 1. (Color online) NiO structure (spins located at the nickel sites): the top view onto the (001) surface shows ferromagnetic rows in $[1 \overline{1} 0]$ direction which couple antiferromagnetically along the [110] direction.

vers have a stiffness of $k \approx 4 \mathrm{kN} / \mathrm{m}$, which enables stable operation at small amplitudes. Small amplitude operation results in a better signal-to-noise ratio, so that the larger noise level at room temperature operation can be offset partially. Despite these efforts, we did not see spin contrast even for very small distances using ferromagnetic and antiferromagnetic tips.

\section{EXPERIMENT}

Nickel oxide crystallizes in the rocksalt structure with a lattice constant of $a_{0}=4.17 \AA$. The spins are localized at the $\mathrm{Ni}$ sites and are pointing to one of the six possible $\langle 1 \overline{2} 1\rangle$ directions. ${ }^{17} \mathrm{NiO}$ is an antiferromagnet with a Néel temperature well above room temperature at $T_{N}=525 \mathrm{~K}$. Within the (111) planes, the spins couple ferromagnetically, and the coupling between neighboring (111) planes is antiferromagnetic (see Fig. 1). The intersection of these planes with the (001) surface yields diagonals with parallel spin alignment, where neighboring lines have opposite spin directions.

The samples used in our experiments were single crystalline blocks of $\mathrm{NiO}$ (SurfaceNet, Rheine, Germany). They were cut to bars of about $2 \times 4 \times 10 \mathrm{~mm}^{3}$ and mounted on a plate to allow sample transfer from ambient conditions to vacuum and in situ sample preparation. A gold layer of about $300 \mathrm{~nm}$ thickness was sputtered onto the samples to support the discharging of the surface right after cleavage. To obtain flat and clean surfaces, the crystals were cleaved in situ with a UHV cleaving device. ${ }^{18}$ All experiments were performed at room temperature at a pressure of $\approx 8 \times 10^{-11}$ mbar. Stable atomic imaging could be achieved for up to four days from the time the cleave was initiated, after that, contamination became visible clearly.

Several estimations of the expected exchange interaction between a magnetic tip and an antiferromagnetic sample surface have been published. First-principle calculations for two magnetic $\mathrm{Fe}(100)$ thin films with a distance in the range of the lattice constant yield $E_{\mathrm{ex}} \approx 10 \mathrm{meV}$ and $F_{\mathrm{ex}} \approx 0.1 \mathrm{nN} .{ }^{19} \mathrm{~A}$ modeling of the $\mathrm{NiO}(001)$ surface interacting with a spin- polarized $\mathrm{H}$ atom (weakly reactive) and a spin-polarized $\mathrm{Fe}$ atom (strongly reactive) finds that the difference in force over opposite spin atoms should be detectable with the AFM for a tip-sample distance smaller than $4 \AA$ or for imaging close to the repulsive regime. ${ }^{20}$ However, at such short distances, the chemical bonding forces can become strong and it was speculated that ion instabilities may become apparent. Elongations of the tip and the sample atomic bonds are no longer negligible and atoms may even become displaced. They may lead to the loss of atomic resolution before the marginal tip-sample distance for detecting the exchange force is reached. Weakly reactive tips are less affected by these instabilities. For bulk $\mathrm{NiO}$, Ködderitzsch et al. ${ }^{21}$ have calculated that the $\mathrm{AF}_{2}$ antiferromagnetic structure displayed in Fig. 1 has a bonding energy that is lower by $116 \mathrm{meV}$ per $\mathrm{Ni}-\mathrm{O}$ atom pair than a ferromagnetic spin arrangement. The Gibbs free energy of formation for $\mathrm{NiO}$ is $211 \mathrm{~kJ} / \mathrm{mol}$ at room temperature, ${ }^{22}$ corresponding to $2.19 \mathrm{eV}$ per $\mathrm{Ni}-\mathrm{O}$ atom pair. Because every ion in $\mathrm{NiO}$ has six neighbors and every bond is shared by two ions, we estimate a bonding energy of $730 \mathrm{meV} /$ bond. Therefore, the use of a NiO tip to probe $\mathrm{NiO}(001)$ promises to provide large spin-dependent contrast where the short-range bonding force varies by $116 \mathrm{meV} / 730 \mathrm{meV}=16 \%$. The range of the exchange forces is expected to be similar to the range of chemical bonds with $\lambda_{\mathrm{ex}} \approx 0.1 \mathrm{~nm}$.

Previous atomically resolved imaging experiments of $\mathrm{NiO}$ surfaces all have parameters in the following ranges: oscillation amplitudes of several nanometers and cantilevers with $k \approx 40 \mathrm{~N} / \mathrm{m}$ oscillating at frequencies of some hundreds of kilohertz. ${ }^{9-16}$ Optimal signal-to-noise ratio is expected for oscillation amplitudes $A \approx \lambda,{ }^{23}$ where $\lambda$ is the range of the interaction that is to be probed. Because of stability requirements, $k \cdot A$ has to exceed a critical value ${ }^{24}$ and a large stiffness is required for stable operation at small amplitudes. For this purpose, the self-sensing quartz cantilever qPlus, ${ }^{25}$ which is based on a commercial tuning fork and can be operated as is with oscillation amplitudes in the range of several angstroms, was modified for operation at even smaller amplitudes. The stiffness of the prongs of the tuning fork is given by $k=E w t^{3} / 4 L^{3}$, where $L, t, w$, and $E$ are the length, the thickness, the width, and Youngs modulus of the prongs, respectively. The modification involved a shortening of the prongs by cutting them with a diamond wire saw, changing $k$ from $1800 \mathrm{~N} / \mathrm{m}$ to $\approx 4000 \mathrm{~N} / \mathrm{m}$ and $f_{0}$ from $\approx 20 \mathrm{kHz}$ to $\approx 40 \mathrm{kHz}$. Stable oscillation at amplitudes of $A \approx 1 \AA$ and below became possible with these "extra stiff" qPlus sensors. Compared to the cantilevers of conventional AFM, $k$ is increased by 100 allowing a decrease of $A$ by a factor of $1 / 100$. As a consequence, additional to the advantage of attenuated long-range background forces, qPlus extra stiff sensors promise to provide an increased frequency shift and thus higher resolution on small scale.

The probe tips are important in AFM. Because of the large size and rigidity of our qPlus force sensors, a wide variety of tips can be mounted. Etched metal tips (e.g., W) as known from scanning tunneling microscopy are standard, but cobalt was chosen as a ferromagnetic tip material. Among the ferromagnetic elements it shows the weakest reactivity which facilitates stable imaging close to the sample surface. The 


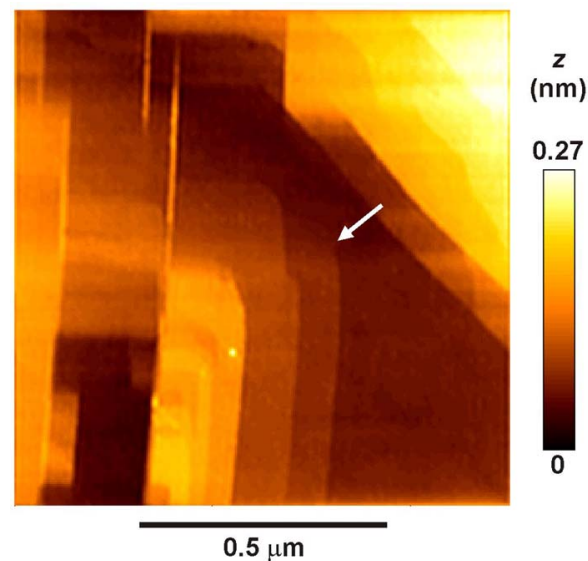

FIG. 2. (Color online) Large scale step structure on $\mathrm{NiO}(001)$ revealed with $\mathrm{FM}-\mathrm{AFM}$ equipped with a $\mathrm{NiO}$ tip $(A \approx 2 \AA, \Delta f$ $=+15 \mathrm{~Hz}$ ). Between wide flat terraces few screw dislocations are visible, such as the one indicated by a white arrow.

etching was performed with a $50 \%$ solution of $\mathrm{HNO}_{3}$. We also prepared antiferromagnetic tips made from $\mathrm{NiO}$ for reasons that are outlined below. $\mathrm{NiO}$ tips were prepared by cleaving larger crystals ex situ and searching for sharply pointed crystallites with sizes of roughly $50 \times 50 \times 250 \mu \mathrm{m} .{ }^{3}$ Annealing by electron bombardment in UHV is difficult for an insulator such as $\mathrm{NiO}$. Therefore, we attempted to clean the tips in situ by scratching along the $(\mathrm{NiO})$ surface.

In typical AFM images of ionic crystals, only one type of ion appears as a protrusion, and the other type is imaged as a depression. It depends on the tip whether $\mathrm{Ni}$ or $\mathrm{O}$ ions are imaged as protrusions in AFM images of $\mathrm{NiO}$ (001). Momida and Oguchi $^{26}$ argued that oxygen atoms appear as bright protrusions when using metal tips because metals react more strongly with oxygen than other metals. However, this issue and the identity of the tip atom and crystallographic environment constitute uncertainties in the image interpretation which will be discussed. Nevertheless, even if the oxygen atoms were imaged bright, contrast variations due to the exchange force are expected because a reduction of the symmetry at surface sites leads to a magnetic moment of the oxygen atoms, too. However, this moment is estimated to be less than $10 \%$ of the one over the nickel sites, so that the exchange effect is expected to be much less pronounced. ${ }^{27}$

\section{RESULTS AND ANALYSIS}

The $\mathrm{NiO}(001)$ surface was investigated with the improved cantilevers that allow stable imaging at oscillation amplitudes as small as $1 \AA$. Cantilevers that carry three different types of tips-nonmagnetic W tips, ferromagnetic Co tips, and antiferromagnetic NiO tips [see Fig. 3(a)] were used in this study. A large scale scan reveals step structures as shown in Fig. 2. The (001) surfaces are not ideal-a few screw dislocations are visible-but flat terraces with a width between 0.05 and $0.5 \mu \mathrm{m}$ provide a good basis for atomic resolution.

Figure 3 shows that atomic contrast on flat and clean
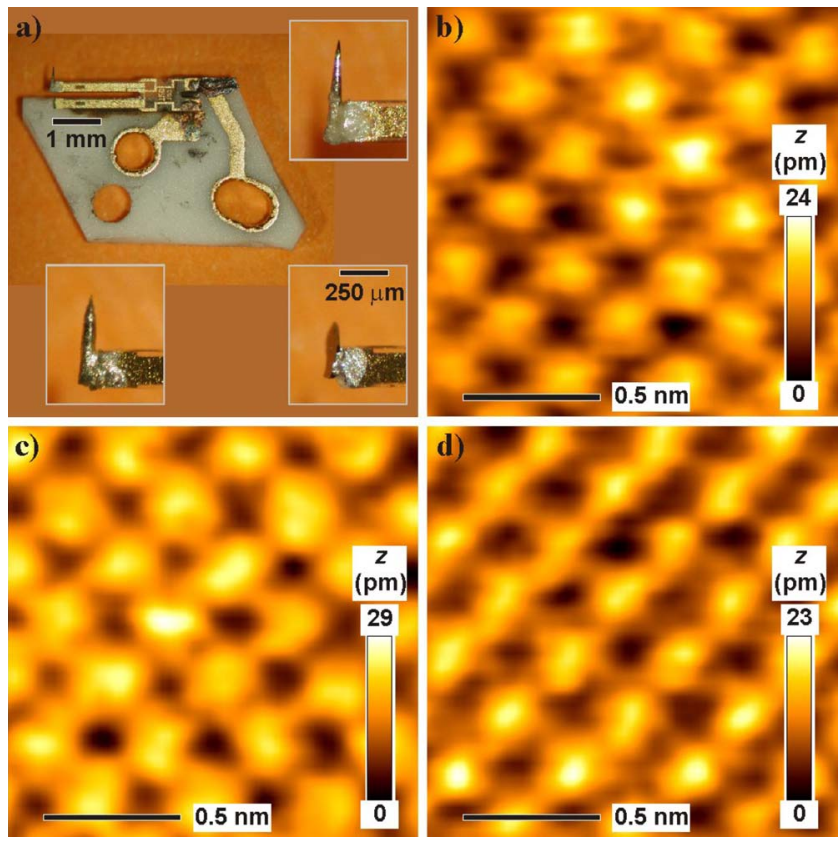

FIG. 3. (Color online) qPlus sensors with tungsten (top), cobalt (bottom left), and nickel oxide (bottom right) tips as shown in (a) allow FM-AFM with atomic resolution on $\mathrm{NiO}(001)$ surfaces; imaging parameters: $A \approx 1 \AA$ and (b) $\Delta f=-20 \mathrm{~Hz}$ (W tip), (c) $\Delta f$ $=-23 \mathrm{~Hz}$ (Co tip), and (d) $\Delta f=-25 \mathrm{~Hz}$ (NiO tip).

$\mathrm{NiO}(001)$ surfaces was obtained with all three kinds of tips [W, Co, and NiO tips in (b), (c), and (d), respectively]. The stiffnesses and eigenfrequencies of the cantilevers that were used are listed in Table I.

The images were acquired at $\Delta f=-20,-23$, and $-25 \mathrm{~Hz}$ with $A \approx 1 \AA$. Therefore, the normalized frequency shift $\gamma$ $=\Delta f k A^{3 / 2} / f_{0}$ was $-2.4,-2.0$, and $-2.3 \mathrm{fN} \sqrt{\mathrm{m}}$, respectively. Neighboring protrusions are spaced by roughly $4 \AA$, indicating that only one sort of atoms is imaged. A corrugation of around $25 \mathrm{pm}$ is observed in these topographical images. The chemical bonding forces responsible for the atomic resolution are assumed to be on the order of $F_{\text {chem }} \approx 1 \mathrm{nN}$ (Ref. 28) -ten times larger than the expected exchange force $\left(F_{\text {ex }}\right.$ $\approx 0.1 \mathrm{nN}$, see above). Contributions of the exchange interaction to the total tip-sample force are expected to cause $10 \%$ of the total atomic corrugation. Because it is not clear whether $\mathrm{Ni}$ or $\mathrm{O}$ appears as a maximum and the exchange corrugation is expected to be maximal on top of $\mathrm{Ni}$, we have to analyze both, maxima and minima, in line profiles.

A detailed investigation of the correlation between the imaging parameters and the corrugation (no images shown here) corroborates the intuitive expectations: Decreasing the

TABLE I. Eigenfrequency and stiffness of the force sensors used in the experiments.

\begin{tabular}{lcc}
\hline \hline & $f_{0}(\mathrm{~Hz})$ & $k(\mathrm{~N} / \mathrm{m})$ \\
\hline W tip & 30675 & 3690 \\
Co tip & 40535 & 3540 \\
NiO tip & 43618 & 4020 \\
\hline \hline
\end{tabular}




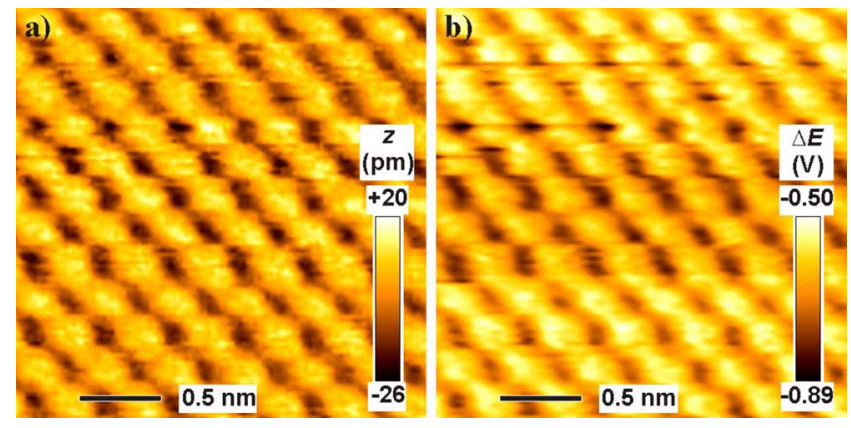

FIG. 4. (Color online) Atomic resolution on $\mathrm{NiO}(001)$ obtained with frequency modulation (FM) AFM in the repulsive mode. The images were taken with a $\mathrm{NiO}$ tip oscillating with $A \approx 1 \AA$ at $\Delta f$ $=+66 \mathrm{~Hz}$. (a) is a topographical picture whereas (b) presents the damping signals.

oscillation amplitude leads to a much clearer resolution and, in addition, to an increased corrugation. Decreasing the set point of the frequency shift $\Delta f$ causes a further approach to the sample surface. Hence, a greater influence of the shortrange forces that lead to the atomic resolution is expected. Indeed the corrugation in associated height profiles of a corresponding series increases with increasing magnitude of the frequency shift set point. These measurements demonstrated that a small amplitude and a large frequency shift are key parameters for obtaining good atomic resolution.

Therefore, we continuously decreased the frequency shift $(\Delta f<0)$ while imaging at small amplitudes. Because of the large stiffness of the modified sensors and the careful choice of the tip material we were able to reach the repulsive regime, where $\Delta f>0$. Atomic resolution of $\mathrm{NiO}(001)$ surfaces with a positive frequency shift was performed, i.e., operation at a distance at or closer than the interatomic distance in bulk NiO. It is important to note that we used $\log |\Delta f|$ as a feedback signal, but we recorded $\Delta f$ as well to confirm the sign of $\Delta f$ (see Ref. 29 for more details). In Fig. 4(a) a topographical image taken with a $\mathrm{NiO}$ tip at $\Delta f=+66 \mathrm{~Hz}$ and $A \approx 1 \AA$, i.e., $\gamma=+2.8 \mathrm{fN} \sqrt{\mathrm{m}}$, is presented. Simultaneously, the dissipation was recorded and the result is shown in Fig. 4(b). The damping is determined from the driving amplitude that is necessary to keep the total energy of the cantilever constant. Variations in the dissipation therefore correspond to changes in the energy of the interactions. ${ }^{30}$ Consequently, influences of the exchange force are expected to be detectable via the attributed changes in energy over adjacent atom sites in the dissipation channel, too. However, estimations yield that the ratio $E_{\text {ex }} / E_{\text {chem }}$ is less favorable than the one of the forces $F_{\text {ex }} / F_{\text {chem }} \approx 1 / 10$.

In total, more than 1000 images with atomic resolution were acquired using different tips in various distance regimes and several channels (topography, frequency shift, damping, and higher harmonics). As shown in Fig. 2, screw dislocations are present on this sample. We expect that screw dislocations alter the spin order, and even if spin alignment between tip and sample may be weak on one region, with all the surface regions that have been scanned there should be one region where spin alignment between tip and sample is sufficient to observe spin contrast. Possible spin order was searched by taking line profiles along the two directions of the diagonals and subsequent comparison. A more sensitive analysis method is offered by fast Fourier transformation (FFT) of the topographical images. The expected antiferromagnetic spin order of $\mathrm{NiO}$ (001) should reveal itself by a peak at half the spatial frequency of the fundamental lattice, thus additional Fourier peaks at $\pm\left(\frac{1}{2 a_{0}}, \frac{1}{2 a_{0}}\right)$ or $\pm\left(\frac{1}{2 a_{0}},-\frac{1}{2 a_{0}}\right)$ should appear as found by Kaiser et al. ${ }^{16}$ The inset in Fig. 5(a) presents the Fourier image of the main topographical image that was acquired with a $\mathrm{NiO}$ tip at $\gamma=-9.0 \mathrm{fN} \sqrt{\mathrm{m}}$ $(\Delta f=-98 \mathrm{~Hz}$ and $A \approx 1 \AA$ ). The expected additional peaks are not present in Fig. 5.

To judge whether spin contrast was observed it is useful to compare the expected spin contrast to the experimental noise of the microscope within the spatial frequency range where the signal is expected. We analyze the FFT data by comparing the height of the main peaks in the FFT image to the noise floor at the spatial frequency where the spin contrast signal is expected. The main peaks at $\left( \pm \frac{1}{a_{0}}, \pm \frac{1}{a_{0}}\right)$ in the FFT image insets in Figs. 5(a) and 5(b) have a height of 17 arbitrary units (a.u.), while the root-mean-square (rms) noise floor integrated over areas $A, A^{\prime}, B$, and $B^{\prime}$ shown in Fig. 5 corresponds at 3.0 and 3.5 a.u. rms, respectively. Since the main peaks refer to a corrugation height of $25 \mathrm{pm}$, the noise in the spatial frequency areas of interest corresponds to $3.5 / 17 \times 25 \mathrm{pm}=5 \mathrm{pm}$. In the Experiment section, we estimated the $16 \%$ effect on the corrugation amounting to a maximum of $0.16 \times 25 \mathrm{pm}=4 \mathrm{pm}$ for perfect spin (anti-) alignment between tip and sample spins. Thus, the signal-tonoise ratio in this experiment is only on the order of 1 for perfect spin alignment and less for an arbitrarily oriented tip.

\section{DISCUSSION}

The fact that the FFT images do not show spin contrast is puzzling. Even at a signal-to-noise ratio less than 1 we would expect to see faint peaks in the FFT images. Calculations have shown that very small tip-sample distances are necessary to observe spin contrast even though tip ion instabilities may result at very small distance. ${ }^{31}$ Here, we have been able to image in the repulsive regime with positive frequency shifts and generally at distances close to the bulk neighbor distance, where optimal spin contrast is expected. ${ }^{26}$ While tips remained stable, we did not observe spin contrast. Stable imaging at a short tip-sample spacing with the ferromagnetic Co tips was possible because of Co's moderate reactivity with $\mathrm{NiO}$. For revealing short-range magnetic forces, another parameter is highly important in addition to the tip-sample spacing, the relative orientation of the interacting spins. Ideally, tip and sample spin are aligned (anti-) parallel, while a misalignment of $60^{\circ}$ is expected to yield half the maximal spin contrast [compare Fig. 6(a)]. Because there are six possible orientations for the spins in the $\mathrm{NiO}$ crystal and because we imaged large areas containing screw dislocations, for a given direction of the tip spin one domain has to exist where the deviation of the relative orientation of the spins is $60^{\circ}$ at most. Considerations of the statistical partition of the spin alignments yield this maximum misalignment angle, too. When imaging $\mathrm{NiO}(001)$ the position on the surface and ac- 

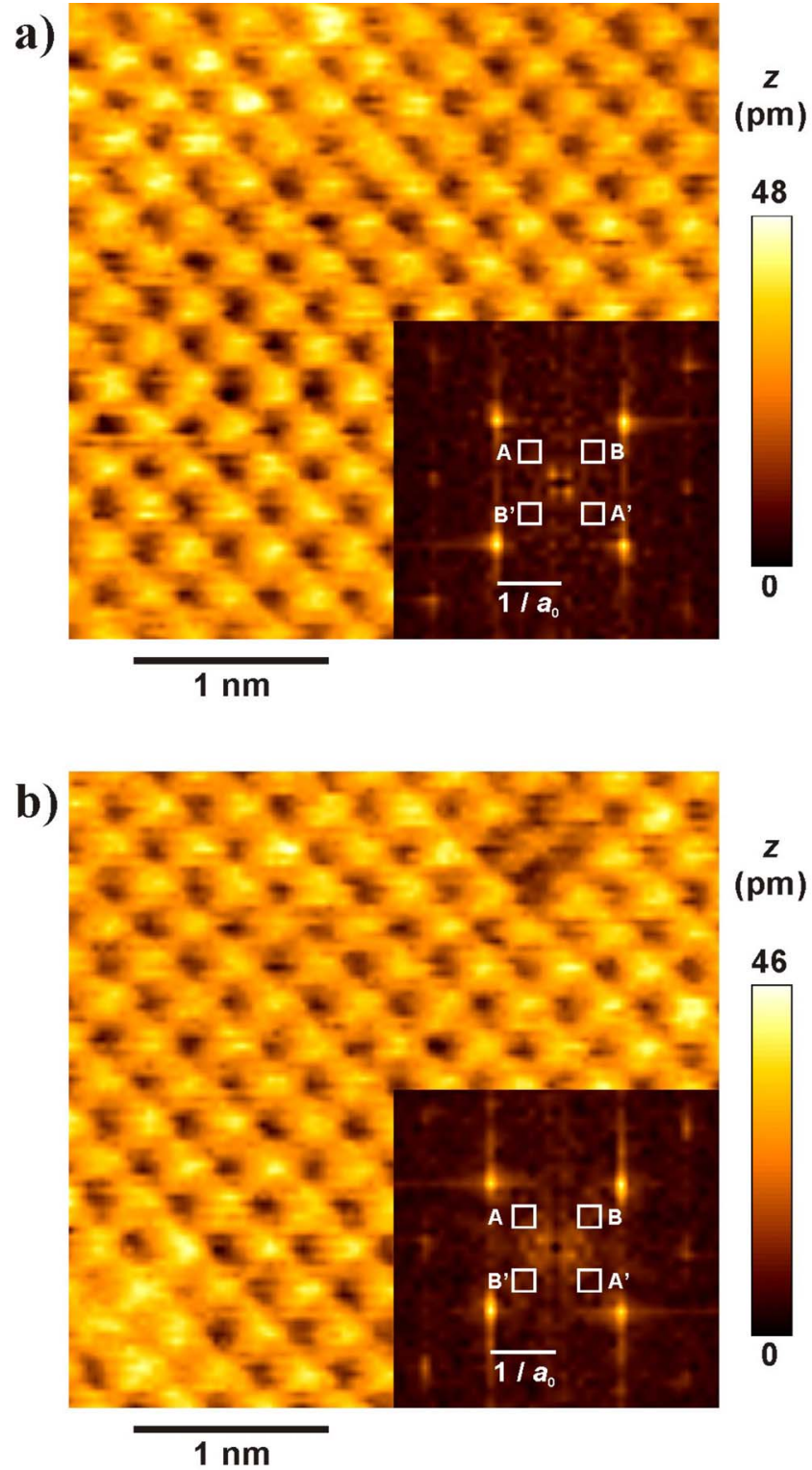

FIG. 5. (Color online) (a) FM-AFM image of a $\mathrm{NiO}(001)$ surface taken with a $\mathrm{NiO}$ tip at $A \approx 1 \AA$ and $\Delta f=-98 \mathrm{~Hz}$ (unfiltered data). The presence of the two defects in the upper right and in the lower left corner shows that true atomic resolution is obtained, i.e., a single tip atom is responsible for imaging. The inset shows the central section of the Fourier transform of the topographical image. A peak at half the spatial frequency of one of the two base peaks would be visible if the contribution of the exchange interaction was larger than instrumental noise (see text). The inset also shows the Fourier transformed image (see text). However, we did not observe a distinguished peak at half the inverse lattice vectors as in Ref. 16. The data presented in (a) and (b) were taken within the same measurement session, a tip change indicated by a glitch and an overall contrast change occurred.

cordingly the investigated magnetic domain was changed multiple times. We have recorded approximately 1000 atomically resolved images of $\mathrm{NiO}(001)$ with various tips and with frequent accidental tip changes that should cause a change in the spin orientation of the tips. Therefore, we assume that favorable spin alignment was present in several cases-at

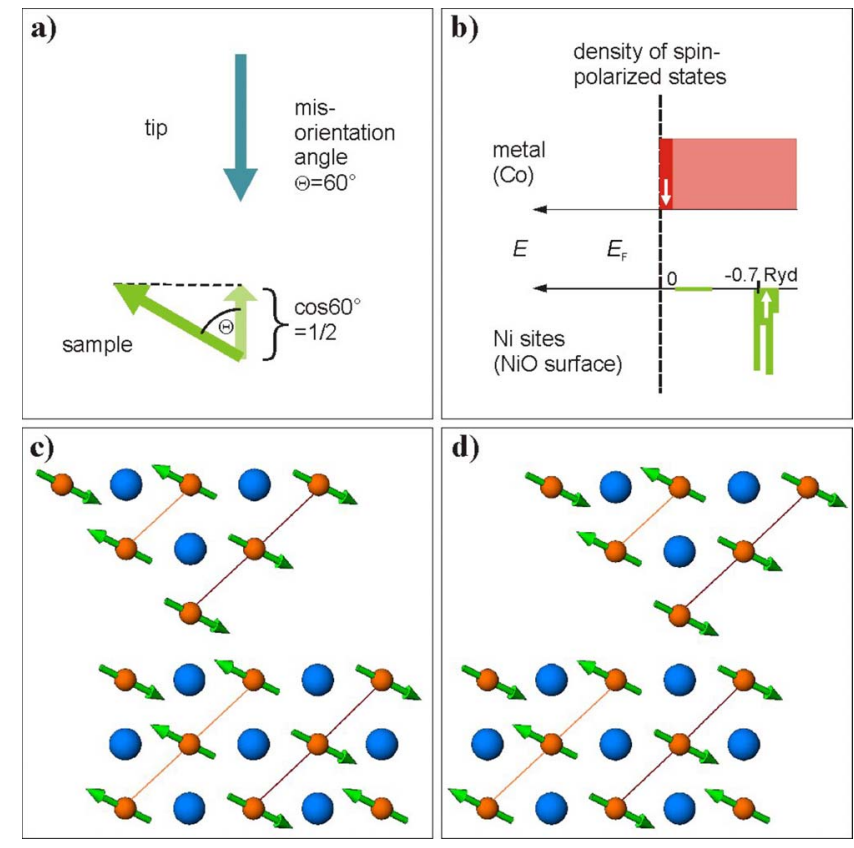

FIG. 6. (Color online) (a) Sketch illustrating the effect of a misalignment between tip and sample spin; (b) comparison of density of spin-polarized states of the $\mathrm{NiO}$ surface and a metal tip revealing the improbability of an interaction; (c) sketch of a $\mathrm{Ni}$ front atom located above an $\mathrm{O}$ sample atom such that spin order is preserved from tip to sample; (d) a Ni front atom also sits on top of an $\mathrm{O}$ sample atom, but the spin order is broken.

least for a limited time as we cannot rule out spin flips of the tip but also within the sample during the scan. None of these many images showed unequivocal signs of spin contrast.

A last consideration regards the tip material. The expected spin contrast originates in the exchange interaction, which is not due to a magnetic dipole-dipole interaction, but due to spin-controlled electrostatic interaction. Exchange interaction can only happen if a bonding orbital between tip and sample evolves, i.e., if an electronic state at a given energy has a large probability amplitude in both tip and sample atoms. The energy of the spin-polarized states located at the $\mathrm{Ni}$ sites of the $\mathrm{NiO}$ surface is about $0.7 \mathrm{Ry}$ below the Fermi level. ${ }^{21}$ Approaching a metal tip-for example, made from cobalt - to the NiO surface, the Fermi levels will match. The spin-polarized states of the metal form a small band below the Fermi level with a bandwidth much smaller than the estimated $10 \mathrm{eV}$ energy difference to the NiO surface. Consequently the formation of a molecular orbital that has a large amplitude on both tip and $\mathrm{NiO}$ sample appears to be unlikely [compare Fig. 6(b)]. Therefore one has to conclude that ferromagnetic metal tips may not be the optimal choice for detecting exchange forces with a $\mathrm{NiO}$ sample. In spinpolarized tunneling, the physical mechanism behind spin contrast is different: the tunneling current is proportional to the spin-dependent density of states in tip and sample, and because electrons are tunneling from tip (sample) states close to the Fermi level into sample (tip) states close to the Fermi level, the energetic equality is automatically fulfilled.

To obtain optimal tip-sample interactions we chose an approach that is conceptually very simple: we manufacture a 
tip of $\mathrm{NiO}$ and measure its interaction with a $\mathrm{NiO}$ surface. If the spin orientation in tip and sample was parallel, the spin orientation depicted in Fig. 6(c), where the spin order continues from tip to sample, is energetically lower than the one shown in Fig. 6(d), where the spin order is broken. It follows that the force in case 6(c) is larger than in 6(d). Spin contrast is expected to become visible no matter whether the tip atom is $\mathrm{Ni}$ or $\mathrm{O}$, because the spin order could be continued in sequence in either case and spins between $\mathrm{Ni}$ ions couple through superexchange in $\mathrm{NiO} .{ }^{32}$

\section{SUMMARY AND OUTLOOK}

In summary, in Fourier space images of $\mathrm{NiO}$ (001), no evidence for spin contrast was found, despite the use of $\mathrm{NiO}$ tips and maximization of the sensitivity to short-range forces by adapting a small amplitude scheme. Spin contrast was also not detectable when using magnetized Co tips, and we have provided a qualitative explanation by arguing that the energy of the spin-polarized states in Co tips and $\mathrm{Ni}$ ions on $\mathrm{NiO}$ does not match. This argument may explain why clear spin contrast has not been observed in very low noise experiments of groups conducted at low temperatures. It is expected that the antiferromagnetic spin order of $\mathrm{NiO}(001)$ is fully developed at room temperature, ${ }^{21}$ but the tip atom of a sharp $\mathrm{NiO}$ crystallite may require much lower temperatures to develop spin order than the bulk. Therefore, we anticipate that the spin contrast signal will become stronger at low temperatures. Repeating this experiment at low temperatures with $\mathrm{NiO}$ tips will result in lower noise, such that spin order not only shows in Fourier images, but in real space images as well. Also, tip preparation can be improved. It would be beneficial to cleave the tips in situ in ultrahigh vacuum. The use of amplitudes in angstrom and subangstrom regimes was only possible by utilizing force sensors with a stiffness on the order of $4 \mathrm{kN} / \mathrm{m}$. Due to a careful choice of tip material, a small tip-sample distance could be realized without losing atomic contrast. These are essential requirements for detecting the extreme short-range exchange interaction between a magnetic tip and the antiferromagnetic sample surface. For the future, we plan to perform these measurements at low temperatures and to utilize advanced tip preparation methods such as in situ tip cleaving. The use of tips made from the same material as the sample has proven to be very successful. This concept may be transferable to other systems, expanding the conceptual beauty of break-junction experiments ${ }^{33}$ to three dimensional imaging.

\section{ACKNOWLEDGMENTS}

We thank T. Eguchi, Ch. Schiller, and M. Breitschaft for helpful discussions and support. This work is supported by the Bundesministerium für Bildung und Forschung (Project No. EKM13N6918) and by the European Union (Nanoxide). *franz.giessibl@physik.uni-regensburg.de

${ }^{1}$ G. Baym, Lectures on Quantum Mechanics (W.A. Benjamin, New York, 1969).

${ }^{2}$ M. Bode, Rep. Prog. Phys. 66, 523 (2003).

${ }^{3}$ S. Heinze, M. Bode, A. Kubetzka, O. Pietzsch, X. Nie, S. Blügel, and R. Wiesendanger, Science 288, 1805 (2000).

${ }^{4}$ A. J. Heinrich, J. A. Gupta, C. P. Lutz, and D. M. Eigler, Science 306, 466 (2004).

${ }^{5}$ G. Binnig, C. F. Quate, and Ch. Gerber, Phys. Rev. Lett. 56, 930 (1986)

${ }^{6}$ K. Nakamura, T. Oguchi, H. Hasegawa, K. Sueoka, K. Hayakawa, and K. Mukasa, Jpn. J. Appl. Phys., Part 1 37, 6575 (1998).

${ }^{7}$ M. Imada, A. Fujimori, and Y. Tokura, Rev. Mod. Phys. 70, 1039 (1998).

${ }^{8}$ M. R. Castell, P. L. Wincott, N. G. Condon, C. Muggelberg, G. Thornton, S. L. Dudarev, A. P. Sutton, and G. A. D. Briggs, Phys. Rev. B 55, 7859 (1997).

${ }^{9}$ H. Hosoi, K. Sueoka, K. Hayakawa, and K. Mukasa, Appl. Surf. Sci. 157, 218 (2000)

${ }^{10}$ H. Hosoi, M. Kimura, K. Hayakawa, K. Sueoka, and K. Mukasa, Appl. Phys. A: Mater. Sci. Process. 72, S23 (2001).

${ }^{11}$ H. Hosoi, K. Sueoka, and K. Mukasa, Nanotechnology 15, 505 (2004).

${ }^{12}$ W. Allers, S. Langkat, and R. Wiesendanger, Appl. Phys. A: Mater. Sci. Process. 72, S27 (2001).

${ }^{13}$ H. Hölscher, S. M. Langkat, A. Schwarz, and R. Wiesendanger, Appl. Phys. Lett. 81, 4428 (2002).

${ }^{14}$ S. M. Langkat, H. Hölscher, A. Schwarz, and R. Wiesendanger, Surf. Sci. 527, 12 (2003).
${ }^{15}$ R. Hoffmann, M. A. Lantz, H. J. Hug, P. J. A. van Schendel, P. Kappenberger, S. Martin, A. Baratoff, and H.-J. Guntherodt, Phys. Rev. B 67, 085402 (2003).

${ }^{16}$ U. Kaiser, A. Schwarz, and R. Wiesendanger, Nature (London) 446, 552 (2007).

${ }^{17}$ F. U. Hillebrecht, H. Ohldag, N. B. Weber, C. Bethke, U. Mick, M. Weiss, and J. Bahrdt, Phys. Rev. Lett. 86, 3419 (2001).

${ }^{18}$ M. Schmid, A. Renner, and F. J. Giessibl, Rev. Sci. Instrum. 77, 036101 (2006).

${ }^{19}$ K. Nakamura, H. Hasegawa, T. Oguchi, K. Sueoka, K. Hayakawa, and K. Mukasa, Phys. Rev. B 56, 3218 (1997).

${ }^{20}$ A. S. Foster and A. L. Shluger, Surf. Sci. 490, 211 (2001).

${ }^{21}$ D. Ködderitzsch, W. Hergert, W. M. Temmerman, Z. Szotek, A. Ernst, and H. Winter, Phys. Rev. B 66, 064434 (2002).

${ }^{22}$ CRC Handbook of Chemistry and Physics, 77 th ed., edited by D. R. Lide (CRC, Boca Raton, FL, 1996).

${ }^{23}$ F. J. Giessibl, AIP Conf. Proc. 696, 60 (2003).

${ }^{24}$ F. J. Giessibl, Rev. Mod. Phys. 75, 949 (2003).

${ }^{25}$ F. J. Giessibl and H. Bielefeldt, Phys. Rev. B 61, 9968 (2000).

${ }^{26}$ H. Momida and T. Oguchi, Surf. Sci. 590, 42 (2005).

${ }^{27}$ H. Momida and T. Oguchi, J. Phys. Soc. Jpn. 72, 588 (2003).

${ }^{28}$ F. J. Giessibl, Mater. Today 8, 32 (2005).

${ }^{29}$ F. J. Giessibl and M. Reichling, Nanotechnology 16, S118 (2005).

${ }^{30}$ R. García and R. Pérez, Surf. Sci. Rep. 47, 197 (2002).

${ }^{31}$ A. S. Foster and A. L. Shluger, Surf. Sci. 490, 211 (2001).

${ }^{32}$ P. W. Anderson, Phys. Rev. 79, 350 (1950).

${ }^{33}$ N. Agrait, A. Yeyati, and J. van Ruitenbeek, Phys. Rep. 377, 81 (2003). 